neuropathies is essential as recent treatment trials show a remission rate of up to $40 \%$.

Aims Compare retrospective data on clinical, investigational and treatment factors in patients who have ceased IVIg with patients who have failed a cessation trial.

Methods 15 patients who successfully suspended IVIg infusions were compared with 15 in whom decreasing or stopping IVIg was unsuccessful.

Results 30 patients (12 with CIDP and 3 with MMN in both groups) were diagnosed 39.5 months from onset of symptoms in the successful group vs. 40.7 months in the unsuccessful group $(p=0.953)$. There was a significant difference in the summed upper limb sensory amplitudes on electrophysiology prior to starting IVIg between the patients with CIDP (17.4 mV vs. $9.8 \mathrm{mV} \mathrm{p}=0.007)$. There was no difference in the average doses between the groups. A successful cessation trial was attempted at a mean of 60.5 months post starting treatment, compared with 60 months in the unsuccessful patients.

Conclusion There is a need for objective biomarker to measure disease activity because other than one neurophysiology marker, other factors did not help predict a successful cessation trial of IVIg.

\section{ENDOVASCULAR CLOT RETRIEVAL (ECR) IN THE ELDERLY. FOR BETTER OR WORSE IN THE REAL WORLD?}

${ }^{1}$ Stacey K Jankelowitz ${ }^{*}$ ' ${ }^{2}$ yllie Tastula, ${ }^{2}$ Nicola Mitchell, ${ }^{2}$ Patrick Tang, ${ }^{3}$ Tim Ang, ${ }^{4}$ David Brunacci, ${ }^{4}$ Geoffrey Parker, ${ }^{3}$ Stephen Winters, ${ }^{4}$ Johnny Wong, ${ }^{4}$ Rodney Allen, ${ }^{2} J o h n$ Worthington. ${ }^{1}$ Royal Prince Alfred Hospital and University of Sydney, Camperdown, NSW, Australia; ${ }^{2}$ Neurosciences, RPAH, Sydney, NSW, Australia; ${ }^{3}$ Neurosciences and Radiology, RPAH, Sydney, NSW, Australia; ${ }^{4}$ Radiology, RPAH, Sydney, NSW, Australia

10.1136/jnnp-2019-anzan.75

\section{Introduction}

Across multicentre trials ECR is safe and effective in octogenarians. Despite RCT evidence elderly patients may be denied ECR due to perceived poor risk-benefit. We examine impact of age on ECR outcomes and outcomes in transcatheter aortic valve implantation (TAVI) cases (where stroke risk is high), in a real world setting.

Methods We analysed 311 consecutive ECR cases between 2016 and 2019 in 10 year age bands for ECR outcomes including 90 day mRS and mortality. Impact of premorbid function (mRS), NIHSS, recorded co-morbidities, and aetiology was assessed. TAVI case outcomes were examined.

Results Thirty one percent of ECR outcome cases were over 79 years of age; 90 day mortality was $34 \%$; $25 \%$ had a 90 day mRS 0-2. Early NIHSS improvement was 5. Ninety-day mortality and mRS 0-2 for 10-19 (n=3), 20-29 (n=2), 30$39(n=4), 40-49 \quad(n=23), 50-59(n=27), 60-69 \quad(n=69), 70-$ $79(\mathrm{n}=84), 80-89 \quad(\mathrm{n}=62)$ and $90-99 \quad(\mathrm{n}=11)$ years were 0 and $100 \%, 0$ and 100\%, 33 and 67\%, 4 and 78\%, 15 and $52 \%, 13$ and 49\%, 17 and 33\%, 24 and $2 \%$ and 55 and $18 \%$, respectively. There was $9 \%$ lost to follow-up.

Six TAVI cases had a NIHSS of $8-20$ and pre-morbid $\mathrm{mRS}<3$, four with mRS 0. Mean 24 hour NIHSS improvement was 8 .

Conclusion Without age exclusions older patients had worse unadjusted outcomes. However, patients over 79 years had clinically important early improvement in NIHSS score and ninety day outcomes were comparable to favourable RCT data and TAVI patients also had early improvement.

\section{CLADRIBINE TABLETS WERE ASSOCIATED WITH RAPID ONSET OF IMPROVEMENTS IN MRI OUTCOMES IN THE ORACLE-MS TRIAL}

${ }^{1}$ Alan Gillett* ${ }^{2}$ Susan Scarberry, ${ }^{3}$ Doris Damian, ${ }^{3}$ Yann Hyvert, ${ }^{3}$ Fernando Dangond, ${ }^{3}$ Lori Lebson, ${ }^{4}$ Thomas Leist. ${ }^{1} E M D$ Serono Inc, Mississauga, ON, Canada; ${ }^{2}$ Sanford Health Multiple Sclerosis Center, Fargo, ND, USA; ${ }^{3}$ EMD Serono, Inc, Billerica, MA, USA; ${ }^{4}$ Division of Clinical Neuroimmunology, Thomas Jefferson University, Jefferson Medical College, Philadelphia, PA, USA

\subsection{6/jnnp-2019-anzan.76}

Introduction In ORACLE-MS (616 subjects with a first demyelinating event at high risk of converting to multiple sclerosis), cladribine tablets (CT) $10 \mathrm{mg}(3.5 \mathrm{mg} / \mathrm{kg}$ or $5.25 \mathrm{mg} / \mathrm{kg}$ cumulative dose over 2 years) significantly delayed the timeto-conversion to clinically definite multiple sclerosis (CDMS), and reduced new/persisting T1 gadolinium-enhancing (T1 Gd + ), new/enlarged or active T2 and combined unique active (CUA) lesion number. Here, the timing of CT effect is evaluated.

Methods MRI scans were performed at screening and every 12 weeks, for non-converting CDMS subjects. MRI-based endpoints were analyzed using analysis of covariance (ANCOVA) and negative binomial models. The temporal effects of the first yearly treatment course of CT and placebo on T1 Gd+, active T2, and CUA lesions were evaluated.

Results 96 weeks: the reduction in mean $\mathrm{T} 1 \mathrm{Gd}+$, active $\mathrm{T} 2$, or CUA lesion number per patient per scan was nominally significantly greater for CT versus placebo $(p<0.0001)$. Early change in $\mathrm{Gd}+$ lesion volume (at Week 13) from baseline was CT, $-155.73 \mathrm{~mm}^{3}$; placebo, $-14.76 \mathrm{~mm}^{3}$. Comparatively larger reductions in mean active $\mathrm{T} 2$ and CUA lesion numbers with CT at Week 13 versus placebo were observed (active T2: CT, -1.25; placebo, -1.43; CUA: CT, -1.56; placebo, -2.41). The mean number of $\mathrm{T} 1 \mathrm{Gd}+$ lesions at 13 weeks following CT was 0.37 versus 1.0 with placebo.

Conclusions MRI data from ORACLE-MS subjects suggest the first yearly treatment course of CT has a rapid onset of action, with beneficial treatment effects on active lesion number and volume evident by Week 13 .

\section{BREAKING THE CYCLE OF CHRONIC DAILY HEADACHE WITH A LOW-DOSE SUBCUTANEOUS LIGNOCAINE AND KETAMINE INFUSION}

${ }^{1}$ Christopher JF Rofe, ${ }^{2,1}$ Raymond Garrick, ${ }^{3,4}$ David Burke, ${ }^{2,1,5}$ Bruce J Brew, ${ }^{2,1,3}$ Susan E Tomlinson*. 'University of Notre Dame, Sydney, NSW, Australia; '2Department of Neurology, St Vincent's Hospital, Sydney, NSW, Australia; ${ }^{3}$ Department of Medicine, Central Clinical School, University of Sydney, Sydney, NSW, Australia; ${ }^{4}$ Department of Neurology, Royal Prince Alfred Hospital, Camperdown, Sydney, NSW, Australia; ${ }^{5}$ Department of Medicine, University of NSW, Sydney, NSW, Australia

\subsection{6/jnnp-2019-anzan.77}

Introduction Management of chronic migraine includes correcting analgesic rebound headache and implementing suitable medication for prevention and acute episodes. However, in many cases this management paradigm oversimplifies the complexity of chronic migraine, particularly the entrenched central pathways that perpetuate chronic migraine. Intravenous 